\title{
Disruption of serine/threonine protein phosphatase 5 inhibits tumorigenesis of urinary bladder cancer cells
}

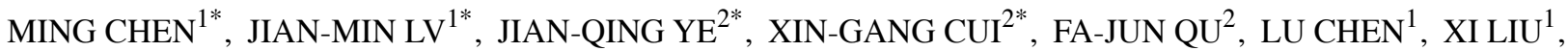 \\ XIU-WU PAN ${ }^{1}$, LIN LI ${ }^{1}$, HAI HUANG ${ }^{1}$, QI-WEI YANG ${ }^{1}$, JIE CHEN $^{1}$, LIN-HUI WANG ${ }^{1}$, \\ $\mathrm{YI} \mathrm{GAO}^{1}$ and DAN-FENG $\mathrm{XU}^{3}$ \\ ${ }^{1}$ Department of Urinary Surgery, Changzheng Hospital, Second Military Medical University, Shanghai 200003; \\ ${ }^{2}$ Department of Urinary Surgery, Third Affiliated Hospital, Second Military Medical University, Shanghai 201805; \\ ${ }^{3}$ Department of Urinary Surgery, Ruijin Hospital, Shanghai Jiaotong University \\ School of Medicine, Shanghai 200025, P.R. China
}

Received January 20, 2017; Accepted March 20, 2017

DOI: 10.3892/ijo.2017.3997

\begin{abstract}
Serine/threonine protein phosphatase 5 (PPP5C) is a member of the protein serine/threonine phosphatase family and has been shown to participate in multiple signaling cascades and tumor progression. We found that PPP5C was highly expressed in bladder cancer tissues compared to normal urothelial tissues, and positively correlated to tumor stages through ONCOMINE microarray data mining. Knockdown of PPP5C via a lentivirus-mediated short hairpin RNA (shRNA) markedly inhibited cell proliferation and colony formation. Flow cytometric analysis showed that PPP5C-deficient T24 and BT5637 bladder cancer cells were arrested in G0/G1 phase and induced apoptosis. In addition, tumor growth was inhibited in vivo in a xenograft nude mouse model. Further studies indicated that knockdown of PPP5C downregulated c-myc and CDK4, whereas upregulated p27, BAD and Beclin1. These results suggest that PPP5C is associated with bladder cancer (BCa) and plays an oncogenic role in the development and progression of bladder cancer.
\end{abstract}

Correspondence to: Dr Dan-Feng Xu, Department of Urinary Surgery, Ruijin Hospital, Shanghai Jiaotong University School of Medicine, Shanghai 200025, P.R. China

E-mail: danfengxu_urology@163.com

Dr Yi Gao, Department of Urinary Surgery, Changzheng Hospital, Second Military Medical University, Shanghai 200003, P.R. China E-mail: gaoyismmu@163.com

*Contributed equally

Abbreviations: GAPDH, glyceraldehyde 3-phosphate dehydrogenase; GFP, green fluorescence protein; MOI, multiplicity of infection; MTT, methylthiazole tetrazolium; PI, propidium iodide; PPP5C, serine/threonine protein phosphatase 5; shRNA, short hairpin RNA; $\mathrm{BCa}$, bladder cancer

Key words: PPP5C, bladder cancer, tumorigenesis, shRNA

\section{Introduction}

Bladder cancer ( $\mathrm{BCa}$ ) is a common malignant tumor in the urinary tract, ranking the first in urologic tumors and twelfth in all cancers among the Chinese populations (1). $\mathrm{BCa}$ is diagnosed yearly in estimated 429,800 patients, of whom about 160,000 succumb to death (2). Other than age, a series of environmental factors contributes to the onset of $\mathrm{BCa}$, which emphasizes the importance of prevention of the disease. Besides, because of the recurrent feature of bladder cancer needing repeated treatment and lifetime surveillance, thus imposing tremendous economic burdens on both society and families. It is therefore urgent to identify molecular candidates involved in the disease and targets for gene therapy.

Protein phosphorylation and dephosphorylation not only affect cell life in multiple ways but play an important part during cancer progression (3). Serine/threonine protein phosphatase 5 (PPP5C) as a member of the protein serine/ threonine phosphatase family is ubiquitously expressed in mammalian cells (4). Several studies have demonstrated that PPP5C is associated with human cancers, among which liver and breast cancers are the most studied $(5,6)$. Moreover, constitutive expression of PPP5C also accelerates tumor growth in mice (7). Despite the discovery of the role of PPP5C in the development and progression of other cancers, whether PPP5C participates in $\mathrm{BCa}$ remains unknown.

As a powerful tool in identifying the function of candidate molecules, RNA interference (RNAi) system has been utilized in cancer gene therapy $(8,9)$. Thus, the present study explored the biological function of PPP5C in bladder cancer using ONCOMINE microarray datasets analysis and lentivirusmediated shRNA system.

\section{Materials and methods}

Cell culture. Human bladder cancer cell lines T24 and BT5637, and human embryonic kidney cell line 293T were purchased from the Cell Bank of the Chinese Academy of Sciences (Shanghai, China). BT5637 cells were cultured in RPMI-1640 medium (HyClone Laboratories, Inc., Logan, 
UT, USA) supplemented with $10 \%$ fetal bovine serum (FBS; Gibco, Carlsbad, CA, USA). T-24 cells were cultured in McCoy's 5A medium (Sigma-Aldrich, St. Louis, MO, USA) supplemented with 10\% FBS (Gibco). 293T cells were cultured in Dulbecco's modified Eagle's medium (DMEM; HyClone Laboratories) supplemented with $10 \%$ FBS. Cells were maintained at $37^{\circ} \mathrm{C}$ in a humidified incubator with a constant air flow of $5 \% \mathrm{CO}_{2}$.

Construction of lentivirus. Small interfering RNA (siRNA) targeting PPP5C (NC_000019.10) (5'-GAGACAGAGAAGAT TACAGTACTCGAGTACTGTAATCTTCTCTGTCTCTTT TT-3') and the non-silencing control sequence (5'-CTAG CCCGGTTCTCCGAACGTGTCACGTATCTCGAGATAC GTGACACGTTCGGAGAATTTTTTTAAT-3') was transformed into short hairpin RNA (shRNA) and cloned into pFH-L green fluorescent protein (GFP) vector (Shanghai Hollybio, Shanghai, China) by double digestion with NheI and $P a c \mathrm{I}$. The generated plasmid shPPP5C-pFH-L or shConpFH-L, together with two pHelper plasmids pVSVG-I and pCMV $\triangle$ R8.92 (Shanghai Hollybio) was transfected into 293T cells. After 72-h transfection, the lentiviruses (Lv-shPPP5C or Lv-shCon) in the culture medium were collected and purified by ultracentrifugation and the titer of each lentivirus was determined.

Cell infection of lentivirus. T24 and BT5637 cells were seeded in 6 -well plates with a concentration of $5 \times 10^{4}$ and $4 \times 10^{4}$ respectively, and then infected with lentivirus Lv-shPPP5C or Lv-shCon at a multiplicity of infection (MOI) of 25 and 35, respectively. The percentage of GFP-positive cells represented the infection efficiency.

Quantitative real-time PCR. After 120-h infection with lentivirus, T24 and BT5637 cells were collected and total RNA was extracted with TRIzol reagent (Invitrogen, Carlsbad, CA, USA). Then, $2 \mu \mathrm{g}$ total RNA was reverse transcribed with Oligo (dT) primer and the M-MLV Reverse Transcriptase (Promega, Madison, WI, USA), according to the manufacturer's instructions. The complementary DNA was used for real-time PCR and a final volume of $20 \mu \mathrm{l}$ was adopted as follows: $10 \mu \mathrm{l} 2 \mathrm{X}$ SYBR Premix Ex Taq (Takara, Dalian, China), $0.8 \mu$ f forward

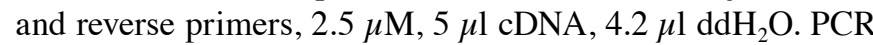
and data collection were performed on CFX Connect ${ }^{\mathrm{TM}}$ RealTime PCR detection system (Takara) and data analysis was operated with the $2^{-\Delta \Delta \mathrm{Ct}}$ method normalized to the endogenous control $\beta$-actin. Primers used in real-time PCR are as follows: $\beta$-actin forward, GTGGACATCCGCAAAGAC and $\beta$-actin reverse, AAAGGGTGTAACGCAACTA; PPP5C forward, GGTGAGGTGAAGGCCAAGTA and PPP5C reverse, TGT GGATCTGACCAGAGCAG.

Western blot assay. One hundred and twenty hours after infection, T24 and BT5637 cells were collected and lysed in 2X SDS lysis buffer (100 mM Tris-HCl, pH 6.8, $10 \mathrm{mM}$ EDTA, 4\% SDS, $10 \%$ glycine). Cell lysates were separated on SDS-PAGE gels and transferred onto polyvinylidene difluoride membranes. The membranes were blocked in TBST buffer with $5 \%$ milk. Then, the membranes were incubated with the following primary antibodies: PPP5C
(\#:11715-1-AP; Proteintech Group, Chicago, IL, USA), CDK4 (\#:11026-1-AP; Proteintech Group), c-Myc (\#:sc-40; Santa Cruz Biotechnology, Santa Cruz, CA, USA), p27 (\#:3686; Cell Signaling Technology, Danvers, MA, USA), BAD (\#:104351-AP; Proteintech Group), Beclin1 (\#:3495; Cell Signaling Technology) and GAPDH (\#:10494-1-AP; Proteintech Group) for $12 \mathrm{~h}$ at $4^{\circ} \mathrm{C}$. After that, horseradish peroxidase conjugated goat anti-rabbit IgG (\#:Sc-2054; Santa Cruz Biotechnology) was applied and incubated for $1 \mathrm{~h}$ at $25^{\circ} \mathrm{C}$. The dilution of each antibody was employed in accordance with the manufacturer's guidelines. The signals of proteins were detected with enhanced chemiluminescence.

Methylthiazoltetrazolium (MTT) cell proliferation assay. BT5637 cells $3 \times 10^{3}$ and $2.5 \times 10^{3}$ T24 cells were cultured in 96-well plates for 1, 2, 3, 4 and 5 days, respectively, after infection with Lv-shCon and Lv-shPPP5C lentivirus for 72 h. 3-(4,5-Dimethylthiazol-2-yl)-2,5-diphenyl tetrazolium bromide (MTT; Sigma-Aldrich) was applied to the cells and after 4-h incubation, the supernatant was discarded. Cells were incubated with acidic isopropanol (10\% SDS, 5\% isopropanol and $0.01 \mathrm{~mol} / 1 \mathrm{HCl}$ ) for $12 \mathrm{~h}$ at $37^{\circ} \mathrm{C}$. The absorbance was detected at $595 \mathrm{~nm}$ using a microplate reader. Each group had five duplicate and the experiment was performed in triplicate.

Colony formation assay. Five hundred T24 cells and BT5637 cells were seeded in 6-well plates and infected with the indicated lentivirus for $72 \mathrm{~h}$. The supernatant was then discarded and culture medium was replaced every 3 days. Subsequently, cells were cultured for another 8 days, fixed with $4 \%$ paraformaldehyde and stained with purple crystals. The number of colonies was analyzed statistically. The experiment was performed three times and each group had three repetitions.

Cell cycle analysis. T24 $\left(1 \times 10^{5}\right)$ and BT5637 cells were seeded in 6-cm dishes and infected with Lv-shCon and Lv-shPPP5C lentivirus for 3 days, followed by 40 -h culture without the virus. The cells were then collected and fixed with $75 \%$ ethanol for $24 \mathrm{~h}$ at $4^{\circ} \mathrm{C}$. Propidium iodide (20 mg/ml) (PI; Sigma-Aldrich) was used to treat the cells and DNA content was recorded with a flow cytometer (BD Biosciences, San Jose, CA, USA) according to the manufacturer's instructions. The experiment was performed in triplicate.

Cell apoptosis analysis. T24 $\left(1 \times 10^{5}\right)$ and BT5637 cells were cultured in 6-cm dishes and infected with Lv-shCon and Lv-shPPP5C lentiviruses for 3 days. Then, the cells were reseeded on 6-cm dishes. After 3 days, Annexin V/PI (BD Biosciences) double staining was performed to analyze cell apoposis by flow cytometry. Each experiment was repeated at least three times.

Animal experiments. Twelve six-week-old nude mice were randomly divided into two groups and then were injected subcutaneously in the right flank with $5 \times 10^{6} \mathrm{~T} 24$ cells preinfected with Lv-shCon and Lv-shPPP5C lentiviruses. Twenty days later, the tumor size was measured every three days and calculated using the formula: (length $\mathrm{x}$ width $\left.{ }^{2}\right) / 2$. At the end of the experiment, the mice were euthanized and the tumors were removed from each nude mouse, imaged and weighed. 
Table I. PPP5C expression and patient characteristics of ALS Bladder dataset.

\begin{tabular}{|c|c|c|c|c|}
\hline & \multirow[b]{2}{*}{ No. of patients } & \multicolumn{2}{|c|}{ PPP5C } & \multirow[b]{2}{*}{ P-value } \\
\hline & & Low & High & \\
\hline Overall, n (\%) & $30(100.0)$ & 15 & 15 & \\
\hline Mean patient age, years (range) & $60.4 \pm 7.3$ & $58.9 \pm 6.8$ & $61.9 \pm 7.7$ & $0.277^{\mathrm{a}}$ \\
\hline Bergkvist grade, n (\%) & & & & $1.000^{\mathrm{b}}$ \\
\hline Grade 1 and 2 & 3 & 1 & 2 & \\
\hline Grade 3 and 4 & 27 & 14 & 13 & \\
\hline T stage, n $(\%)$ & & & & $1.000^{\mathrm{b}}$ \\
\hline$\leq \mathrm{T} 2 \mathrm{~b}$ & 3 & 2 & 1 & \\
\hline$\geq \mathrm{T} 3 \mathrm{a}$ & 27 & 13 & 14 & \\
\hline M stage, n (\%) & & & & $0.466^{\mathrm{b}}$ \\
\hline M0 & 15 & 9 & 6 & \\
\hline M1 & 15 & 6 & 9 & \\
\hline Overall survival status, n (\%) & & & & $0.042^{\mathrm{b}}$ \\
\hline Alive & 5 & 5 & 0 & \\
\hline Dead & 25 & 10 & 15 & \\
\hline
\end{tabular}

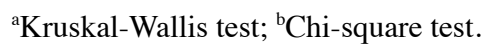

Bioinformatic analysis of the ONCOMINE microarray datasets. The expression level of PPP5C in BCa was analyzed using the ONCOMINE microarray datasets (www.onocomine. org), which contain a great deal of public and published microarray datasets with tens of thousands of samples. By using 'PPP5C', 'BCa' and 'mRNA' as the search terms, 17 datasets were acquired, of which 3 datasets with 7 subunits included PPP5C expression in BCa and normal urothelial tissues. Then, a meta-analysis was carried out to compare the level of the PPP5C between the $\mathrm{BCa}$ and the normal tissue. Besides, we compared the expression of the PPP5C from $\mathrm{T}$ stage and grade malignancy of the $\mathrm{BCa}$ in the Blaveri Bladder 2 and Dyrskjot Bladder datasets. Information on survival time in ALS Bladder was obtained for survival analysis. All data are reported $\log 2$ Median-Centered intensity in the ONCOMINE microarray datasets.

Statistical analysis. Data are presented as mean \pm SD from at least three experiments. Statistical comparison between the Lv-shCon and Lv-shPPP5C was performed using Student's t-test. The PPP5C expression and patient characteristics of the $\mathrm{BCa}$ were analyzed using the Chi-square test in the $\mathrm{BCa}$ tissue microarray. In addition, the Kaplan-Meier analysis was used to evaluate the relation between the prognosis and the PPP5C expression in ALS microarray datasets. The SPSS version 19 (IBM Corp., Armonk, NY, USA) was used to calculate the statistics and $\mathrm{P}<0.05$ was considered statistically significant.

\section{Results}

PPP5C expression of the bladder cancer on the ONCOMINE microarray datasets. To evaluate the expression level of the
PPP5C in BCa, the public online ONCOMINE microarray database was used to perform meta-analysis for evaluation of PPP5C expression in BCa. A total of 3 datasets including 7 groups comparing PPP5C expression between the $\mathrm{BCa}$ and normal urothelial tissue were included in this study, which comprised $343 \mathrm{BCa}$ and 130 normal urothelial tissues. As shown in Fig. 1B, the expression level of PPP5C in BCa was higher than that in the normal tissue in all three datasets. Besides, the results of the meta-analysis of the 7 groups in the 3 datasets also support that the PPP5C was overexpressed in BCa (Fig. 1A), and the difference was statistically significant between $\mathrm{BCa}$ and normal urothelial tissues. Furthermore, the correlation between PPP5C expression and T stage and malignant grade of BCa was analyzed. It was found that PPP5C was highly expressed in $\mathrm{T}$ stages $\geq \mathrm{T} 1$ compared to that in $\mathrm{Ta}$ of BCa (Fig. 1D and E), and in high-grade BCa than that in lowgrade $\mathrm{BCa}$ (Fig. 1F). Patients with low-level PPP5C expression indicated a better prognosis in ALS Bladder microarray dataset (Table I). Similarly, Kaplan-Meier survival analysis of ALS dataset showed that overall survival (OS) was poor in patients with upgraded PPP5C (Fig. 1C).

Lentivirus-mediated interference downregulates PPP5C expression in bladder cancer cells. To determine the role of PPP5C in BCa, a lentivirus-mediated shRNA system was used to silence PPP5C in two human BCa cell lines T24 and BT5637. The cells were infected with Lv-shPPP5C or the control lentivirus Lv-shCon, with an infection efficiency of $>90 \%$ identified by GFP expression.

The mRNA and protein levels of PPP5C in Lv-shPPP5Cinfected T24 and BT5637 was significantly reduced in day 3 and 5 post-transduction, respectively (Fig. 2B-E). These findings suggest that lentivirus-mediated RNAi efficiently 


\section{A \\ Comparison of PPP5C Across 7 Analyses \\ Over-expression}

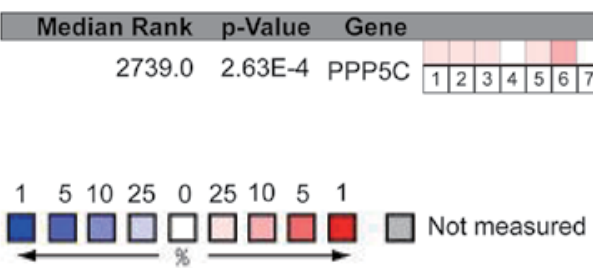

The rank for a gene is the median rank for that gene across each of the analyses

The P-value for a gene is its $\mathrm{P}$-value for the medianranked analysis
Legend

1. Infiltrating Bladder Urothelial Carcinoma vs.Normal Dyrskjot Bladder 3, Cancer Res, 2004

2. Stage Ois Bladder Urothelial Carcinoma vs.Normal Dyrskjot Bladder 3, Cancer Res, 2004

3. Superficial Bladder Cancer vs. Normal Dyrskjot Bladder 3, Cancer Res, 2004

4. Infiltrating Bladder Urothelial Carcinoma vs.Normal Lee Bladder, J Clin Oncol, 2010

5. Superficial Bladder Cancer vs. Normal Lee Bladder, J Clin Oncol, 2010

6. Infiltrating Bladder Urothelial Carcinoma vs. Norma Sanchez-Carbayo Bladder 2, J Clin Oncol, 2006

7. Superficial Bladder Cancer vs. Normal

Sanchez-Carbayo Bladder 2, J Clin Oncol, 2006
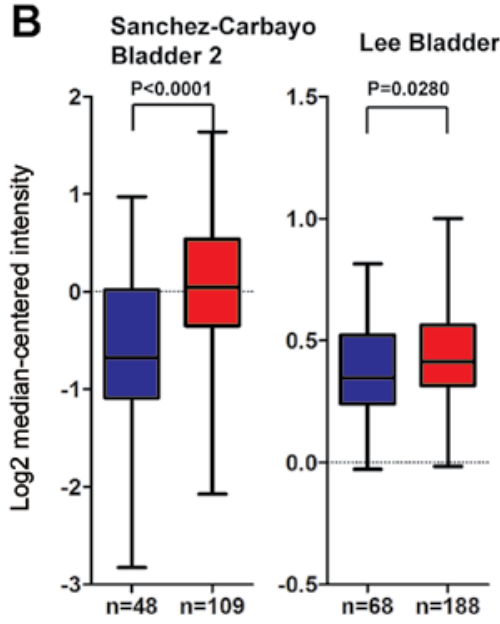

Dyrskjot Bladder 3

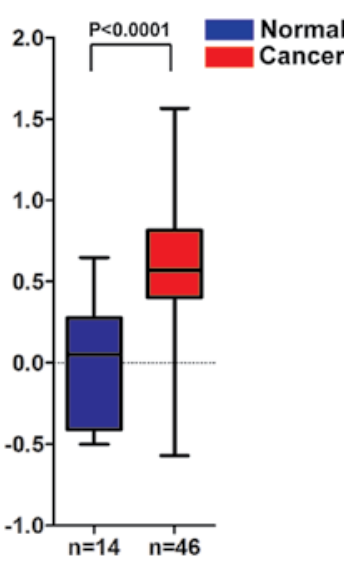

C

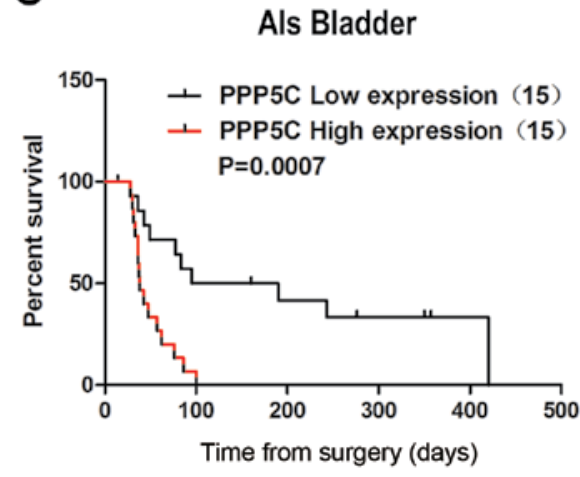

D Blaveri bladder 2

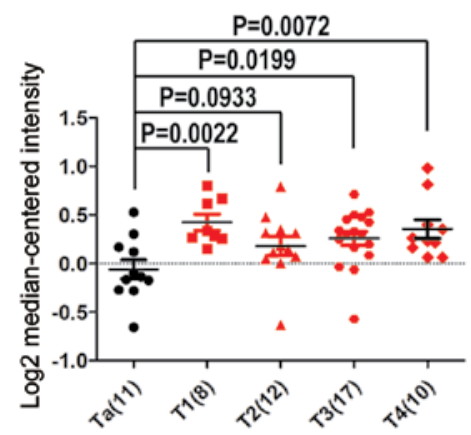

E Dyrskjot Bladder

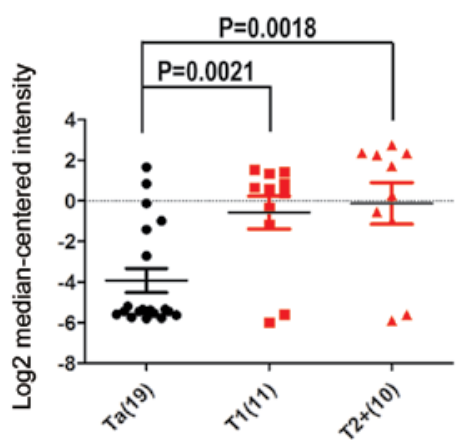

$\mathbf{F}$

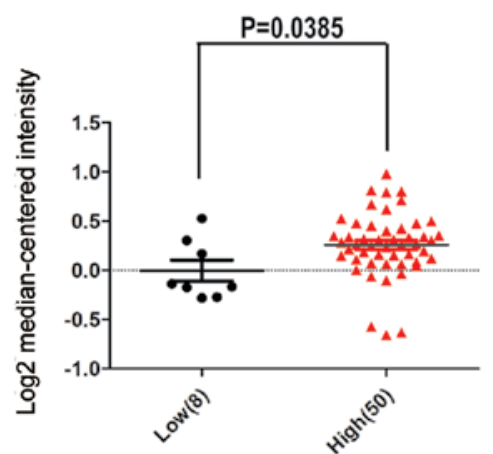

Figure 1. High expression of PPP5C is associated with T stage, malignant degree and prognosis in BCa as analyzed by ONCOMINE microarray database. (A) Meta-analysis of the PPP5C expression between the $\mathrm{BCa}$ and the normal urothelial tissue from 3 microarray datasets containing 7 groups (P<0.001). (B) Each expression difference of the bladder cancer and normal tissue in the Sanchez-Carbayo Bladder 2 dataset $(\mathrm{P}<0.0001)$, Lee $\mathrm{Bladder}$ dataset $(\mathrm{P}=0.028)$, Dyrskjot Bladder 3 ( $\mathrm{P}<0.0001)$. (C) The Kaplan-Meier analysis on the correlation between the PPP5C and the survival using the Als dataset ( $\mathrm{P}=0.0007)$. (D and E) The two microarray datasets (Blaveri bladder 2 and Dyrskjot Bladder) show that the expression of PPP5C in $\mathrm{T}$ stage $\geq \mathrm{T} 1$ was higher than that in Ta. (F) The Blaveri bladder 2 dataset indicated that the expression of PPP5C in BCa of high-grade malignancy was higher than that in BCa of low-grade malignancy $(\mathrm{P}=0.0385)$.

downregulated the endogenous PPP5C expression in urinary BCa cell lines.

Lentivirus-mediated knockdown of PPP5C suppresses the viability and proliferation of bladder cancer cells in vitro. To identify whether PPP5C played a role in tumorigenesis, MTT and colony formation assay were adopted. Compared with Lv-shCon-infected T24 cells, the viability of cells infected with Lv-PPP5C and Lv-shPPP5C was significantly reduced (Fig. 3A and B).

To further elucidate the role of PPP5C in cancer cell proliferation, colonies formed in T24 cells after PPP5C knockdown were analyzed. As shown in Fig. 3C, colony formation in Lv-shPPP5C-infected cells was obviously impaired with respect to morphology and quantity, as compared with Lv-shCon-infected cells. In addition, the number of colonies 


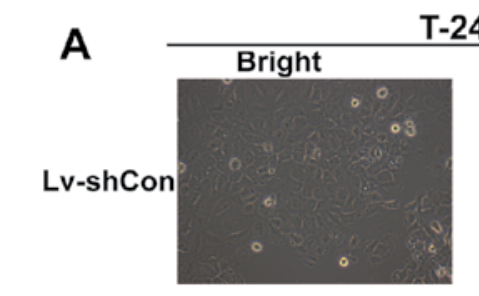

T-24
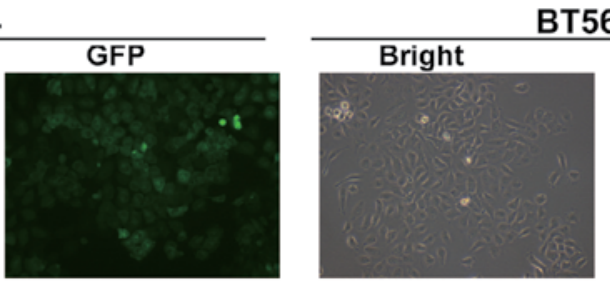

BT5637
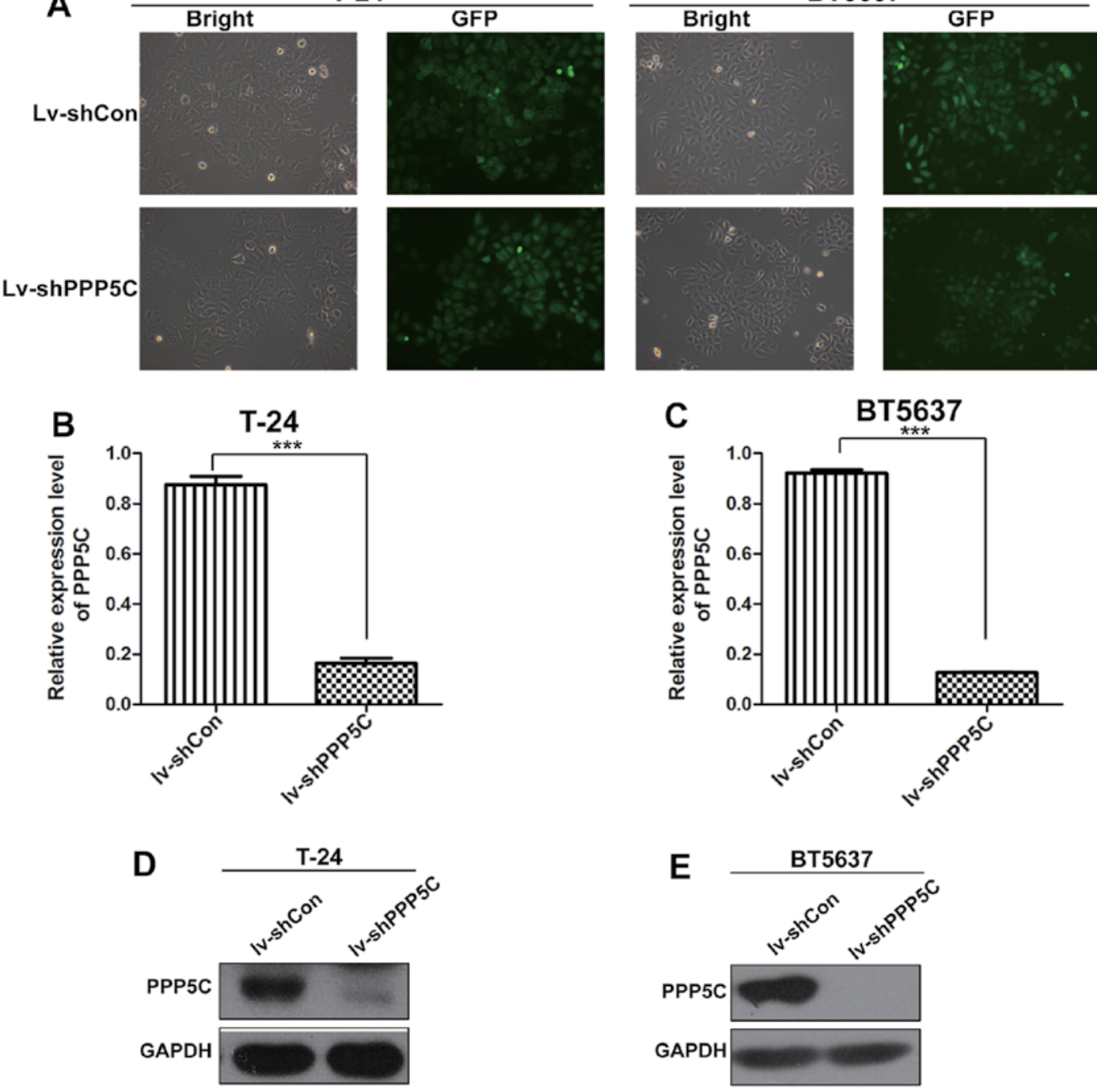

Figure 2. Lentivirus-mediated interference downregulates the expression of PPP5C in urinary bladder cancer cells. (A) T24 and BT5637 cells were examined by fluorescence microscopy after lentivirus infection for $72 \mathrm{~h}$. (B and C) Knock-down efficiency of PPP5C mRNA level in T24 and BT5637 infected by Lv-shPPP5C was determined by real-time qPCR $\left({ }^{* * *} \mathrm{P}<0.001\right)$. (D and E) The protein level of PPP5C was downregulated in T24 and BT5637 cells. Lv-shCon, lentivirus-mediated non-silencing shRNA; Lv-shPPP5C, lentivirus-mediated non-silencing shPPP5C. Data were presented as mean \pm SD from at least three experiments.

in Lv-shPPP5C-infected cells was reduced by nearly $50 \%$ (Fig. 3E). Similar result was also observed in BT5637 cells (Fig. 3D and F). In summary, our data demonstrate that PPP5C expression was of importance for the viability and proliferation of $\mathrm{BCa}$ cells.

Knockdown of PPP5C inhibits bladder cancer cell growth in vivo. To further validate the oncogenic function of PPP5C in vivo, Lv-shPPP5C-infected and Lv-shCon-infected T24 cells were injected subcutaneously to the six-week-old nude mice, and the tumor volume and weight were measured after tumor formation. As shown in Fig. 4, tumor size and weight in LV-shPPP5C-infected mice were significantly reduced as compared with those in mice treated with Lv-shCon-infection. These results further support that PPP5C functions as an oncogene in vivo.

Disruption of PPP5C expression leads to cell cycle arrest of the bladder cancer cells. Tumorigenesis largely depends on accelerated or at least normal cell cycle of cancer cells. Knowing that PPP5C played an important role in BCa cell proliferation, flow cytometric analysis of cell cycle distribution through PI staining to see whether PPP5C participated in the regulation of cancer cell cycle showed that after infection with Lv-shPPP5C, T24 cells were arrested in G0/G1 phase compared with Lv-shCon cells (65.61 \pm 0.86 vs. $56.49 \pm 0.57 \%)$, and the number of cells in $\mathrm{G} 2 / \mathrm{M}$ phase was decreased $(11.54 \pm 0.24$ vs. $19.37 \pm 0.49 \%$ ) (Fig. 5A and B). Similarly, the proportion of BT5637 cells in G1/G0 phase in Lv-shPPP5C group was increased $(74.6 \pm 0.88$ vs. $67.66 \pm 1.74 \%)$ and the number of cells in $\mathrm{G} 2 / \mathrm{M}$ phase was reduced significantly $(4.55 \pm 2.28$ vs. $10.08 \pm 2.03 \%)$. These findings suggest that knockdown of PPP5C could cause T24 and BT5637 cell cycle arrest in G0/G1 phase.

Downregulation of PPP5C expression induces apoptosis of the BCa cells. To further confirm the effect of PPP5C on $\mathrm{BCa}$ cell apoptosis, flow cytometric analysis through Annexin V/PI 
A
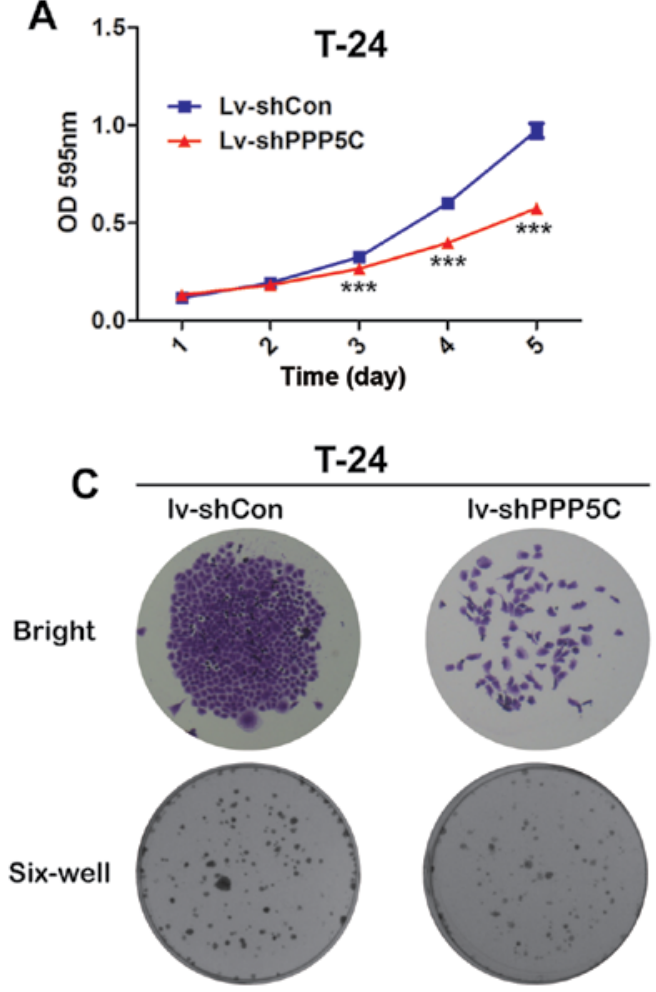

E

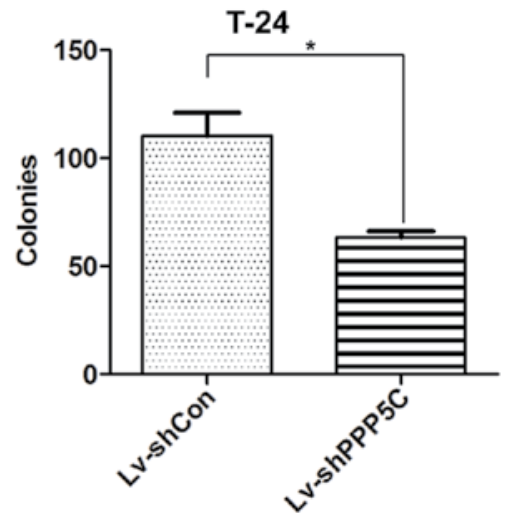

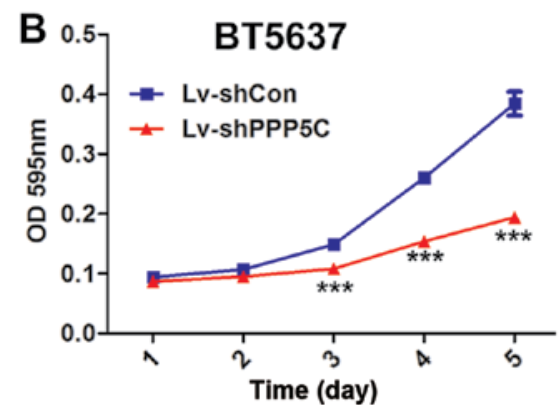

D

BT5637

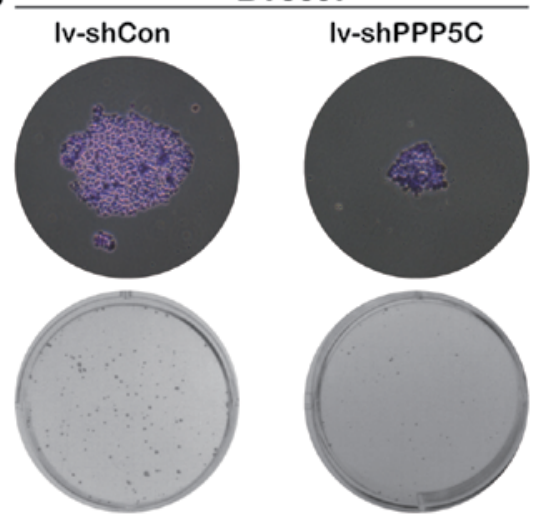

$\mathbf{F}$

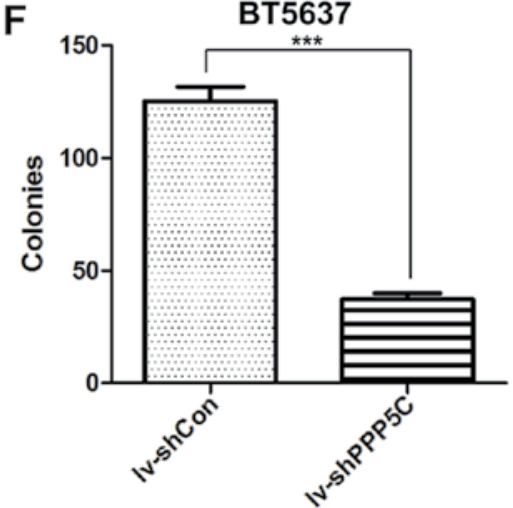

Figure 3. Lentivirus-mediated knockdown of PPP5C suppresses cell viability of urinary bladder cancer cells. (A and B) After infection with Lv-shCon or Lv-shPPP5C, the growth curves of T24 and BT5637 cells were determined by MTT assay. Data are presented as mean \pm SD from at least three experiments $\left({ }^{* * *} \mathrm{P}<0.001\right)$. (C and D) Representative images of T24 and BT5637 cells colony formation under micro- and macro-views after infection with Lv-shCon or Lv-shPPP5C. (E and F) The number of colonies formed in T24 and BT5637 cells treated as in (C and D). Data are presented as mean \pm SD from at least three experiments $\left({ }^{*} \mathrm{P}<0.05,{ }^{* * *} \mathrm{P}<0.001\right)$.

double staining was performed in T24 and BT5637 cells after lentivirus infection. The four domains divided by Annexin V and PI plots represent the different states of cells. Viable cells were located in Annexin $\mathrm{V}^{-} / \mathrm{PI}^{-}$, necrotic cells in Annexin $\mathrm{V}^{-} /$ $\mathrm{PI}^{+}$, early apoptotic cells in Annexin $\mathrm{V}^{+} / \mathrm{PI}^{-}$, and late apoptotic cells in Annexin $\mathrm{V}^{+} / \mathrm{PI}^{+}$(Fig. 6A). As shown in Fig. 6B, early and late apoptosis were increased significantly in lv-shPPP5C as compared with lv-shCon both in T24 and BT5637 cells. Above all, PPP5C silencing promoted apoptosis of BCa cells.

ShRNA-mediated depression of PPP5C regulates the protein level of CDK4, c-Myc, p27, BAD and Beclin1. To further clarify the mechanism of the PPP5C in regulating the biological function of the bladder cancer, the expression of downstream signaling molecule were analyzed by western blot analysis.
The level of CDK4 and c-Myc was decreased whereas the level of p27, BAD and Beclin1 was increased (Fig. 7). These results provide evidence that interfering with the expression of PPP5C could regulate apoptosis, cell cycle and other biological functions.

\section{Discussion}

Compared with other solid cancers, much less is known about the molecular mechanism of BCa. Despite a variety of recent genome-wide profiling studies, there is still no valid strategy for early diagnosis or therapeutic intervention of BCa (10-12). Therefore, the confirmation of critical molecular signatures is badly needed for the effective treatment of the malignancy. 

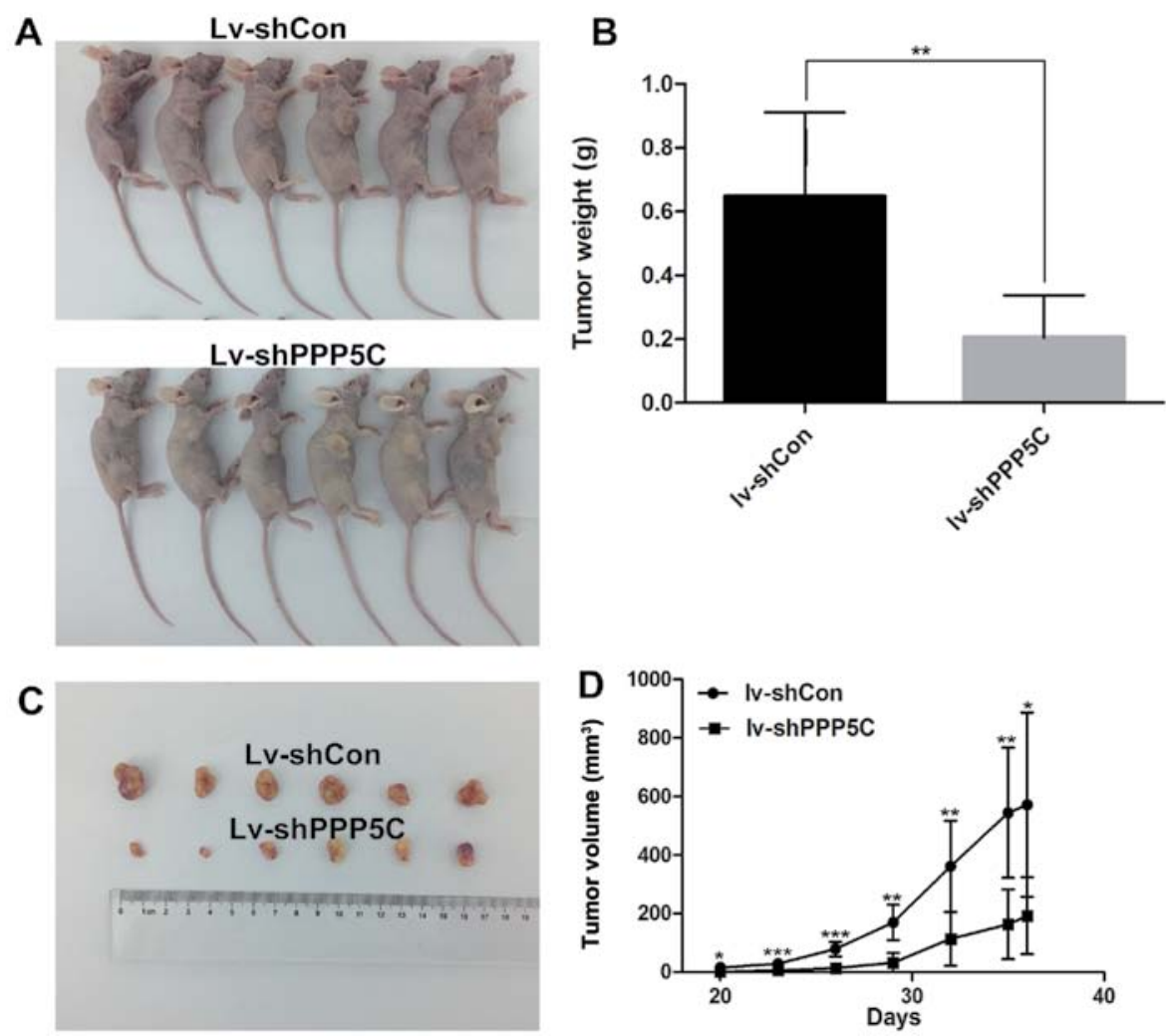

Figure 4. Knock-down of the PPP5C level inhbits the growth of BCa in vivo. (A and C) The subcutaneous xenograft nude mice and the tumor sample were collected and imaged. (B and D) The tumor size and weight were significantly decreased in nude mice infected with lv-shPPP5C-T24 cell ( $\mathrm{n}=6$ ) as compared with those in nude mice infected with lv-shCon-T24 $(\mathrm{n}=6)\left({ }^{*} \mathrm{P}<0.05,{ }^{* *} \mathrm{P}<0.01,{ }^{* * * *} \mathrm{P}<0.001\right)$.

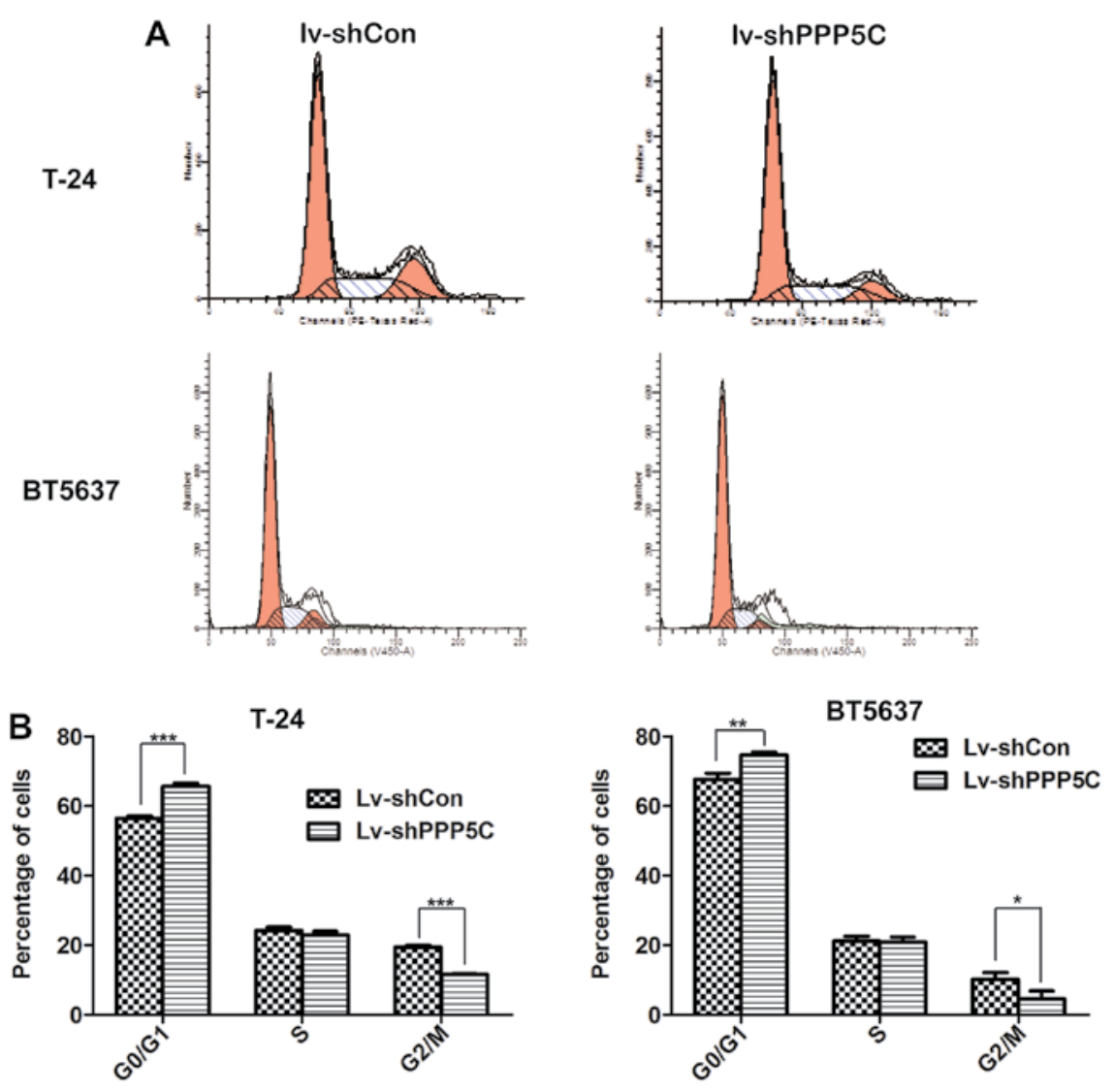

Figure 5. Disruption of PPP5C expression causes cell cycle arrest in bladder cancer cells. (A) Flow cytometric analysis showing cell cycle distribution of T24 and BT5637 cells infected by Lv-shCon or Lv-shPPP5C. (B) Cell percentages in G0/G1, S and G2/M of T24 and BT5637 cells treated as in (A) were analyzed statistically $\left({ }^{*} \mathrm{P}<0.05,{ }^{* *} \mathrm{P}<0.01,{ }^{* * *} \mathrm{P}<0.001\right)$. 
A
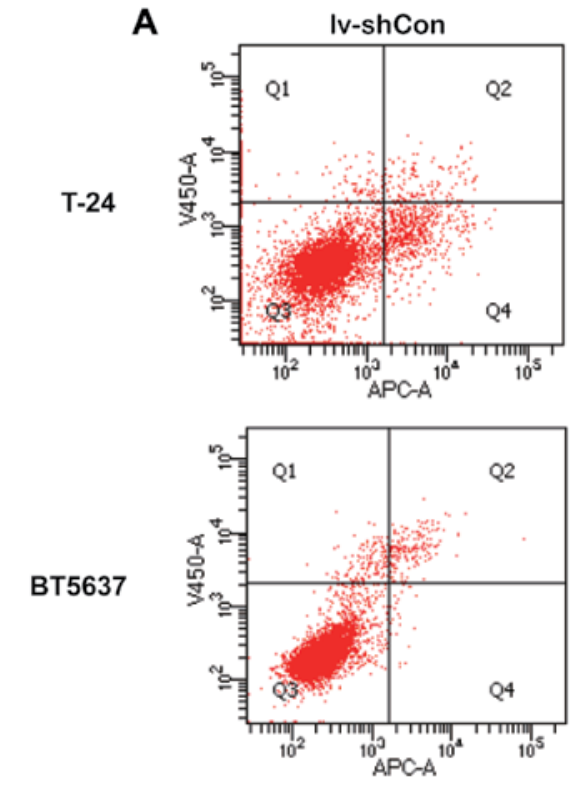

B

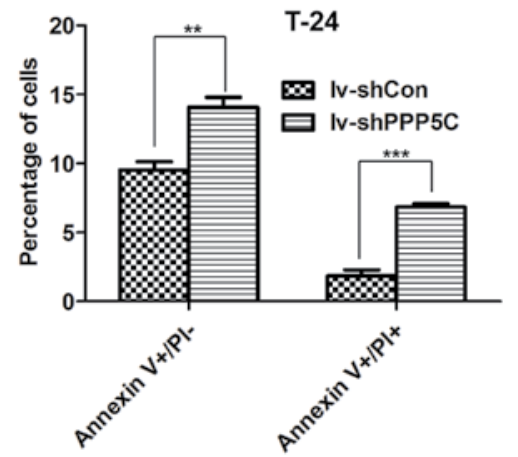

IV-shPPP5C
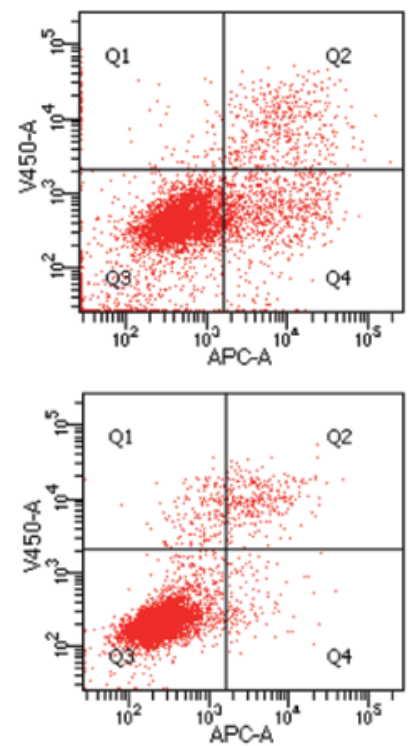

BT5637

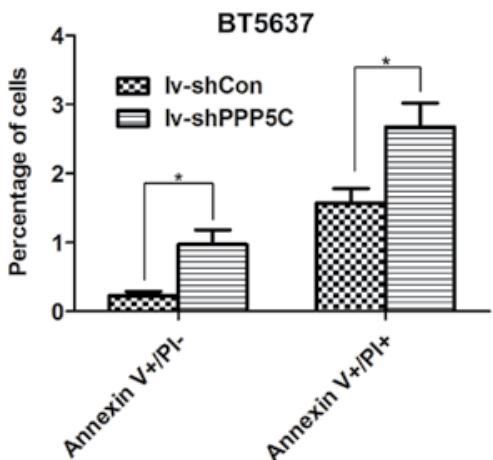

Figure 6. Apoptotic cells are increased after PPP5C silencing. (A) Apoptosis of T24 and BT5637 was analyzed in lv-shCon and lv-shPPP5C groups using flow cytometry and Annexin V/PI staining. (B) Early apoptosis $\left(\mathrm{V}^{+} / \mathrm{PI}\right)$ and late apoptosis $\left(\mathrm{V}^{+} / \mathrm{PI}^{+}\right)$of the $\mathrm{T} 24$ and BT5637 cells were statistically analyzed ("P $<0.05$, $\left.{ }^{* *} \mathrm{P}<0.01,{ }^{* * *} \mathrm{P}<0.001\right)$.

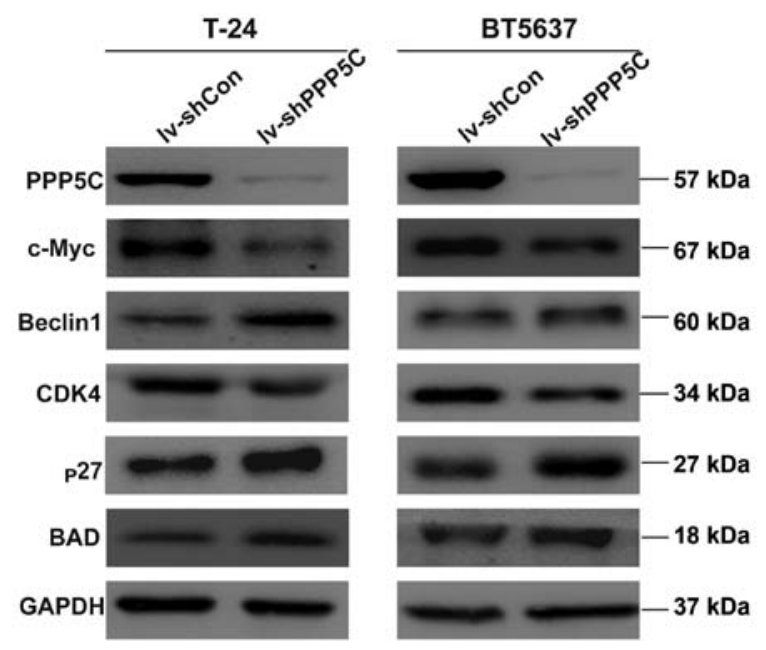

Figure 7. The protein level transformation by western blot analysis. As shown CDK4 and c-Myc were decreased, and BAD, Beclin1 and § were increased after infection with lv-shPPP5C vs. lv-shCon. GAPDH was used as loading control.

PPP5C is a ubiquitously-expressed protein with only one form rather than two or more isoforms of other phosphoprotein phosphatase members $(13,14)$. The biological function of PPP5C remains largely unknown. Some studies have reported its role in cancer development. Elevation of PPP5C has been found both in human cancer tissues and human breast cancer cell line MCF-7 (5). PPP5C is also associated with other cancers such as liver cancer and several other cellular signaling cascades $(6,14,15)$. However, few studies have focused on the role of PPP5C in BCa progression. Given the severe consequences and economic burden brought by $\mathrm{BCa}$, we aimed to find out whether PPP5C was its candidate biomarker and provide a novel therapeutic target for $\mathrm{BCa}$.

The ONCOMINE microarray datasets is a cancer microarray database and integrated data-mining platform (16) that can be used to analyze differential expressions between cancer and paired normal tissues. Besides, the relation between gene expression and various clinical data with respect to pathology, prognosis, metastasis and drug resistance can also be analyzed using the ONCOMINE microarray dataset. Increased numbers of studies have begun utilizing the datasets to validate and explore their research results (17-19). Using the ONCOMINE microarray dataset, the present study found that PPP5C was upregulated in $\mathrm{BCa}$, which was correlated with $\mathrm{T}$ stage, malignant grade and survival of BCa patients (Fig. 1). 
The data that we obtained from the ONCOMINE microarray dataset suggest that PPP5C may play a role in the development and progression of $\mathrm{BCa}$.

To confirm the oncogenic function of PPP5C in $\mathrm{BCa}$, we used a lentivirus-mediated RNA interference system to interfere with the expression of PPP5C in BCa cells. Due to its ability to integrate into the genome of host cells, lentiviruses have been extensively employed as vectors for shRNA expression (22). Even in clinical trials, lentivirus-delivered shRNA have been utilized without notable side-effects (21,22). Lentivirus-mediated knockdown of PPP5C revealed that PPP5C was crucial for the viability and proliferation of bladder cancer in vitro and in vivo.

Cell proliferation is closely related to cell cycle progression. In G0/G1 phase, cells undergo DNA amplification and nutrient accumulation, with no cell differentiation (23). Disturbance of the cell cycle will impair cell proliferation. It was found in the present study that PPP5C knockdown induced cancer cell arrest in G0/G1 phase, with fewer cells in G2/M phase, indicating that PPP5C played a role in regulating bladder cell cycle. Western blot analysis showed that the expression of c-Myc and CDK4 was decreased and the expression of p27 was increased, indicating a relationship between PPP5C and cell cycle arrest. Being the most important subtype of cyclin dependent kinases, CDK4 can guarantee cells to complete DNA replication and pass through the $\mathrm{G} 1$ check point into $\mathrm{S}$ phase of the cell cycle. Besides, c-Myc, a vital transcriptional regulatory factor, also plays an important role in regulating G1 phase via multiple mechanisms including transcriptional activation of CDK4 directly (24). Thus, decrease in CDK4 may be a main reason for G0/G1 phase arrest. In addition, p27 as an important member of the cyclin-dependent kinase inhibitor family can inhibit the activity of the cyclin/CDK complex and negatively regulate the cell cycle (25). Hence, the increased expression of p27 was related to G0/G1 phase arrest.

Although we have demonstrated the biological function of PPP5C in bladder cancer cells, the exact molecular mechanism needs to be further explored. The MAPK (mitogen-activated protein kinases), a conserved family of enzymes, comprising several major components (JNK, P38 and ERK) play an important role in the cellular biological processes, such as proliferation, apoptosis, stress response and metabolism (26-30). In addition, regulation of MAPK cascade phosphorylation plays an important role in its function. Thus, as a kind of the phosphatase, PPP5C may affect the function of MAPK through dephosphorylation, such as regulating the phosphorylation of ERK and JNK by interfering with cell proliferation and apoptosis (17,31-33).

In conclusion, we demonstrated that lentivirus-mediated shRNA targeting PPP5C played a crucial role in viability, proliferation, apoptosis and migration of BCa cells, suggesting that PPP5C may prove to be a potential therapeutic target for the treatment of urinary $\mathrm{BCa}$.

\section{Acknowledgements}

The present study is supported by grants from the National Natural Science Foundation of China for Youths (81502211), the Shanghai Committee of Science and Technology General
Program for Medicine (15ZR413900) and the Shanghai Changzheng Hospital Grant for Youth (2015CZQN05).

\section{References}

1. Chen W, Zheng R, Baade PD, Zhang S, Zeng H, Bray F, Jemal A, Yu XQ and He J: Cancer statistics in China, 2015. CA Cancer J Clin 66: 115-132, 2016.

2. Torre LA, Bray F, Siegel RL, Ferlay J, Lortet-Tieulent J and Jemal A: Global cancer statistics, 2012. CA Cancer J Clin 65: 87-108, 2015.

3. Cohen P: The role of protein phosphorylation in human health and disease. The Sir Hans Krebs Medal Lecture. Eur J Biochem 268: 5001-5010, 2001.

4. Hinds TD Jr and Sánchez ER: Protein phosphatase 5. Int J Biochem Cell Biol 40: 2358-2362, 2008.

5. Golden T, Aragon IV, Rutland B, Tucker JA, Shevde LA, Samant RS, Zhou G, Amable L, Skarra D and Honkanen RE: Elevated levels of Ser/Thr protein phosphatase 5 (PP5) in human breast cancer. Biochim Biophys Acta 1782: 259-270, 2008.

6. Shirato H, Shima H, Nakagama H, Fukuda H, Watanabe Y, Ogawa K, Matsuda Y and Kikuchi K: Expression in hepatomas and chromosomal localization of rat protein phosphatase 5 gene. Int J Oncol 17: 909-912, 2000.

7. Golden T, Aragon IV, Zhou G, Cooper SR, Dean NM and Honkanen RE: Constitutive over expression of serine/threonine protein phosphatase 5 (PP5) augments estrogen-dependent tumor growth in mice. Cancer Lett 215: 95-100, 2004.

8. Ren W, Wang X, Gao L, Li S, Yan X, Zhang J, Huang C, Zhang Y and Zhi K: MiR-21 modulates chemosensitivity of tongue squamous cell carcinoma cells to cisplatin by targeting PDCD4. Mol Cell Biochem 390: 253-262, 2014.

9. Lu J, Luo JH, Pang J, Cao JZ, Wu RH, Tong ZT, Chen W and Xie D: Lentivirus-mediated RNA interference of clusterin enhances the chemosensitivity of EJ bladder cancer cells to epirubicin in vitro. Mol Med Rep 6: 1133-1139, 2012.

10. Peter S, Borkowska E, Drayton RM, Rakhit CP, Noon A, Chen W and Catto JW: Identification of differentially expressed long noncoding RNAs in bladder cancer. Clin Cancer Res 20: 5311-5321, 2014.

11. Matsuda K, Takahashi A, Middlebrooks CD, Obara W, Nasu Y, Inoue K, Tamura K, Yamasaki I, Naya Y, Tanikawa C, et al: Genome-wide association study identified SNP on 15q24 associated with bladder cancer risk in Japanese population. Hum Mol Genet 24: 1177-1184, 2015.

12. Draaken M, Knapp M, Pennimpede T, Schmidt JM, Ebert AK, Rösch W, Stein R, Utsch B, Hirsch K, Boemers TM, et al: Genome-wide association study and meta-analysis identify ISL1 as genome-wide significant susceptibility gene for bladder exstrophy. PLoS Genet 11: e1005024, 2015.

13. Swingle MR, Honkanen RE and Ciszak EM: Structural basis for the catalytic activity of human serine/threonine protein phosphatase-5. J Biol Chem 279: 33992-33999, 2004.

14. Golden T, Swingle M and Honkanen RE: The role of serine/ threonine protein phosphatase type 5 (PP5) in the regulation of stress-induced signaling networks and cancer. Cancer Metastasis Rev 27: 169-178, 2008.

15. Morita K, Saitoh M, Tobiume K, Matsuura H, Enomoto S, Nishitoh $\mathrm{H}$ and Ichijo $\mathrm{H}$ : Negative feedback regulation of ASK1 by protein phosphatase 5 (PP5) in response to oxidative stress. EMBO J 20: 6028-6036, 2001 .

16. Rhodes DR, Yu J, Shanker K, Deshpande N, Varambally R, Ghosh D, Barrette T, Pandey A and Chinnaiyan AM: ONCOMINE: A cancer microarray database and integrated data-mining platform. Neoplasia 6: 1-6, 2004.

17. Malik R, Khan AP, Asangani IA, Cieślik M, Prensner JR, Wang X, Iyer MK, Jiang X, Borkin D, Escara-Wilke J, et al: Targeting the MLL complex in castration-resistant prostate cancer. Nat Med 21: 344-352, 2015.

18. Huang Y, Pan XW, Li L, Chen L, Liu X, Lu JL, Zhu XM, Huang H, Yang QW, Ye JQ, et al: Overexpression of USP39 predicts poor prognosis and promotes tumorigenesis of prostate cancer via promoting EGFR mRNA maturation and transcription elongation. Oncotarget 7: 22016-22030, 2016.

19. Pan XW, Chen L, Hong Y, Xu DF, Liu X, Li L, Huang Y, Cui LM, Gan SS, Yang QW, et al: EIF3D silencing suppresses renal cell carcinoma tumorigenesis via inducing $\mathrm{G} 2 / \mathrm{M}$ arrest through downregulation of Cyclin B1/CDK1 signaling. Int J Oncol 48: 2580-2590, 2016. 
20. ter Brake O, Konstantinova P, Ceylan $M$ and Berkhout $B$ Silencing of HIV-1 with RNA interference: A multiple shRNA approach. Mol Ther 14: 883-892, 2006.

21. Bank A, Dorazio R and Leboulch P: A phase I/II clinical trial of beta-globin gene therapy for beta-thalassemia. Ann NY Acad Sci 1054: 308-316, 2005.

22. Manilla P, Rebello T, Afable C, Lu X, Slepushkin V, Humeau LM, Schonely K, Ni Y, Binder GK, Levine BL, et al: Regulatory considerations for novel gene therapy products: A review of the process leading to the first clinical lentiviral vector. Hum Gene Ther 16: 17-25, 2005.

23. Menon SG and Goswami PC: A redox cycle within the cell cycle: Ring in the old with the new. Oncogene 26: 1101-1109, 2007.

24. Amati B, Alevizopoulos K and Vlach J: Myc and the cell cycle. Front Biosci 3: d250-d268, 1998.

25. Abukhdeir AM and Park BH: P21 and p27: Roles in carcinogenesis and drug resistance. Expert Rev Mol Med 10: e19, 2008.

26. Dickinson RJ and Keyse SM: Diverse physiological functions for dual-specificity MAP kinase phosphatases. J Cell Sci 119: 4607-4615, 2006.

27. Rovida E and Stecca B: Mitogen-activated protein kinases and Hedgehog-GLI signaling in cancer: A crosstalk providing therapeutic opportunities? Semin Cancer Biol 35: 154-167, 2015.
28. Wagner EF and Nebreda AR: Signal integration by JNK and p38 MAPK pathways in cancer development. Nat Rev Cancer 9: 537-549, 2009.

29. Zhang YL and Dong C: MAP kinases in immune responses. Cell Mol Immunol 2: 20-27, 2005.

30. Low HB and Zhang Y: Regulatory Roles of MAPK phosphatases in cancer. Immune Netw 16: 85-98, 2016.

31. Shah BH and Catt KJ: Protein phosphatase 5 as a negative key regulator of Raf-1 activation. Trends Endocrinol Metab 17: 382-384, 2006.

32. von Kriegsheim A, Pitt A, Grindlay GJ, Kolch W and Dhillon AS: Regulation of the Raf-MEK-ERK pathway by protein phosphatase 5. Nat Cell Biol 8: 1011-1016, 2006.

33. Han X, Xu B, Beevers CS, Odaka Y, Chen L, Liu L, Luo Y, Zhou H, Chen W, Shen T, et al: Curcumin inhibits protein phosphatases $2 \mathrm{~A}$ and 5 , leading to activation of mitogen-activated protein kinases and death in tumor cells. Carcinogenesis 33: 868-875, 2012. 